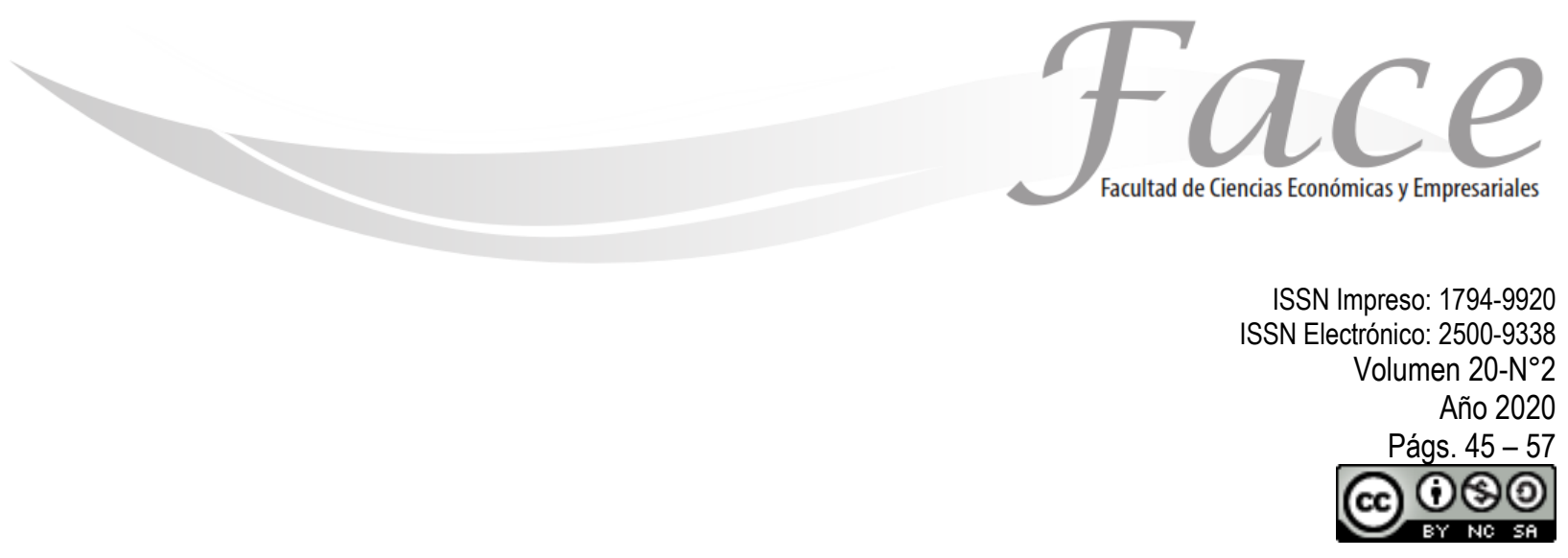

\title{
COSTOS OCULTOS RELACIONADOS CON DISFUNCIONAMIENTOS EN LA IMPLEMENTACIÓN DE LA ESTRATEGIA DE UNA EMPRESA RESTAURANTERA
}

\author{
Salvador Guerrero Moreno * \\ Enlace ORCID: https://orcid.org/0000-0002-4886-7549 \\ Martín Aubert Hernández Calzada ** \\ Enlace ORCID: https://orcid.org/0000-0001-7225-7831 \\ Dra. Carla Carolina Pérez Hernández * \\ Enlace ORCID: https://orcid.org/0000-0001-8286-8775
}

Fecha de Recepción: Septiembre 18 de 2020

Fecha de Aprobación: Diciembre 20 de 2020

\section{Resumen:}

La gestión socioeconómica estimula la adaptación de las empresas a su entorno, promoviendo un funcionamiento eficiente al equilibrar los factores sociales y económicos para el logro de sus objetivos. El Modelo Socioeconómico permite conocer el impacto económico de los disfuncionamientos a través de la estimación de sus costos ocultos.

El artículo presenta un diagnóstico cualimétrico realizado a una pequeña empresa mexicana de la industria restaurantera con el objetivo de conocer los costos ocultos relacionados con la implementación de la estrategia, mediante la metodología de investigación-intervención, para elaborar un Plan de Acciones Estratégicas Internas y Externas como propuesta de mejora.

Palabras clave: Costos ocultos, Disfuncionamientos, Modelo Socioeconómico, Restaurantes, Estrategia.

* Maestro en Administración. Profesor por asignatura del Instituto de Ciencias Económico Administrativas, Universidad Autónoma del Estado de Hidalgo, México. Contacto: salvador querrero@uaeh.edu.mx

** Doctor en Nuevas Tendencias en Dirección de Empresas. Profesor-Investigador, Instituto de Ciencias Económico Administrativas, Universidad Autónoma del Estado de Hidalgo, México. Contacto: martinh@uaeh.edu.mx

** Doctora en Ciencias Económico Administrativas. Profesora-Investigadora, Instituto de Ciencias Económico Administrativas, Universidad Autónoma del Estado de Hidalgo, México. Contacto: carla_perez@uaeh.edu.mx 


\title{
HIDDEN COSTS RELATED TO DYSFUNCTIONS IN THE STRATEGY IMPLEMENTATION OF A RESTAURANT COMPANY
}

\begin{abstract}
:
Socioeconomic management stimulates the adaptation of companies to their environment, promoting their efficient operation by balancing social and economic factors to achieve their objectives. The Socioeconomic Model makes it possible to know the economic impact of dysfunctions through the estimation of hidden costs.

The article presents a qualimetric diagnosis carried out on a small Mexican company in the restaurant industry with the objective of knowing the hidden costs related to the strategy implementation, through the researchintervention methodology, to develop an Internal and External Strategic Actions Plan as a proposal for improvement.
\end{abstract}

Keywords: Hidden costs, Dysfunctions, Socioeconomic Model, Restaurants, Strategy.

\section{CUSTOS OCULTOS RELACIONADOS ÀS DESFUNÇÕES NA IMPLEMENTAÇÃO DA ESTRATÉGIA DE RESTAURANTE}

Resumo:

A gestão socioeconômica estimula a adaptação das empresas ao seu ambiente, promovendo uma operação eficiente por meio do equilíbrio entre os fatores sociais e econômicos para atingir seus objetivos. O Modelo Socioeconômico permite conhecer o impacto econômico das disfunções por meio da estimativa de seus custos ocultos.

O artigo apresenta um diagnóstico qualimétrico realizado em uma pequena empresa mexicana do setor de restaurantes com o objetivo de conhecer os custos ocultos relacionados à implementação da estratégia, através da metodologia de pesquisa-intervenção, para desenvolver um Plano de Ações Estratégicas Internas e Externas como uma proposta de melhoria.

Palavras-chave: Custos ocultos, Disfunções, Modelo Socioeconômico, Restaurantes, Estratégia. 


\section{INTRODUCCIÓN:}

La falta de consolidación de las empresas en México obedece a una amplia variedad de factores que afectan su desarrollo y crecimiento en un mercado agresivo y cada vez más cambiante. Durante el censo económico del Instituto Nacional de Estadística y Geografía (INEGI, 2020), se identificó que el $94 \%$ de los establecimientos son micro, pequeñas y medianas empresas (Mipymes), de las cuales casi el $20 \%$ pertenecen a servicios privados no financieros, donde se ubican los restaurantes.

La misma referencia pone en evidencia elementos que afectan directamente la continuidad de las Mipymes mexicanas en el tiempo como: capacitación, pues sólo el $29 \%$ la llevan a cabo (2.4\% en el caso particular de las microempresas), rotación de personal, cuya tasa promedio es del $24 \%$ en un año, o la tendencia generalizada a disminuir las remuneraciones por persona. Asimismo, se puede considerar que los principales problemas que manifestaron se deben a la inseguridad, el elevado monto de los gastos y los compromisos fiscales (INEGI, 2020).

En cuanto a la industria restaurantera, esta se define como "los servicios de preparación de alimentos y bebidas para su consumo inmediato en el mismo establecimiento o fuera de éste" (INEGI, 2016), la cual integra elementos tangibles en los alimentos y bebidas, junto con el servicio que representa el intangible del negocio. Según la Cámara Nacional de la Industria de Restaurantes y Alimentos Condimentados (CANIRAC, 2016), uno de cada diez comercios registrados formalmente corresponde a este giro, consolidándose como el segundo referente dentro del sector de los servicios en generación de empleo.

De acuerdo con lo anterior, cada día cobra mayor relevancia para las empresas la generación de estrategias efectivas que les permitan alcanzar de manera constante y sostenida el logro de sus objetivos, orientando apropiadamente sus esfuerzos y recursos. En este sentido, el Modelo Socioeconómico (MSE) propuesto por Henri Savall en los años setentas, se postula como un referente que reconoce y busca integrar de manera armónica los componentes sociales con los económicos, bajo un ambiente sano de trabajo, mediante la gestión de comportamientos y la orientación de las estructuras (Savall y Zardet, 2008).

La gestión socioeconómica, busca dotar de herramientas e información a las empresas y organizaciones para adaptarse al acelerado ritmo del entorno. Su hipótesis marca que todos los actores que participan pueden ejercer un poder no oficial en la organización, afectando el cambio a través de los disfuncionamientos (diferencias entre lo planeado y lo obtenido), lo que acarrea costos y desempeños ocultos (Savall et al., 2008. p.164) que impactan en los resultados obtenidos.

El objetivo del estudio es identificar los disfuncionamientos relacionados con la implementación de la estrategia de una pequeña empresa restaurantera, mediante el MSE, para proponer estrategias de mejora que permitan reducir la incidencia de los costos ocultos.

\section{MARCO TEORICO:}

La fundamentación teórica principal del estudio se basa en el MSE propuesto por Henri Savall, en el cual se buscan integrar los componentes sociales con los económicos de una organización de manera armónica bajo un buen ambiente laboral, entendiendo que si una empresa logra dominar su operación tiene mayores posibilidades de tener éxito en su entorno. El modelo se convierte en una herramienta útil para consultoría e investigación que busca diseñar soluciones factibles para resolver la problemática real de la empresa (García, 2016), mediante un proceso cognitivo interactivo entre los actores de la unidad objeto de estudio y los consultores-investigadores (Pérez, 2012).Comenzando por definir una empresa, el Artículo 16 de la Ley Federal del Trabajo en México dice que es "una unidad económica de producción o distribución de bienes o servicios" (LFT, 2019). 
Asimismo, González y Ganaza (2015) la conciben como una unidad económica que "proporciona bienes o servicios a cambio de un precio que le permite la reposición de recursos empleados y la consecución de objetivos predeterminados, estando su utilidad en la capacidad que tiene de satisfacer una necesidad" ( $p$. 17).

El propósito del modelo está orientado al diseño de una metodología de gestión socioeconómica que integre conceptos de investigación y consultoría empresarial con un enfoque tanto teórico como práctico, entendiendo la importancia de la participación de todos los actores involucrados en la organización para generar una mejora continua (Savall et al., 2008), procurando la coordinación entre instrumentos con orientación financiera y social (Parra y Peña, 2014). resalta que ninguna "aportaba resultados operativos en si", puesto que no integran los sistemas no oficiales con los oficiales.

Con la intención de desarrollar y probar la gestión socioeconómica, se ha experimentado el método en más de cuatro continentes a través de programas de investigación apoyados por el Instituto de SocioEconomía de las Empresas y de las Organizaciones (ISEOR), fundado en 1975, donde se ha trabajado en identificar y evaluar los costos ocultos de los disfuncionamientos, tal cómo se observa en la Hipótesis Fundamental del Análisis Socioeconómico (Figura 1) que reconoce el poder no oficial de los colaboradores en respuesta a las estructuras organizacionales (Savall y Zardet, 2009).

Figura 1.

Hipótesis Fundamental del Análisis Socioeconómico

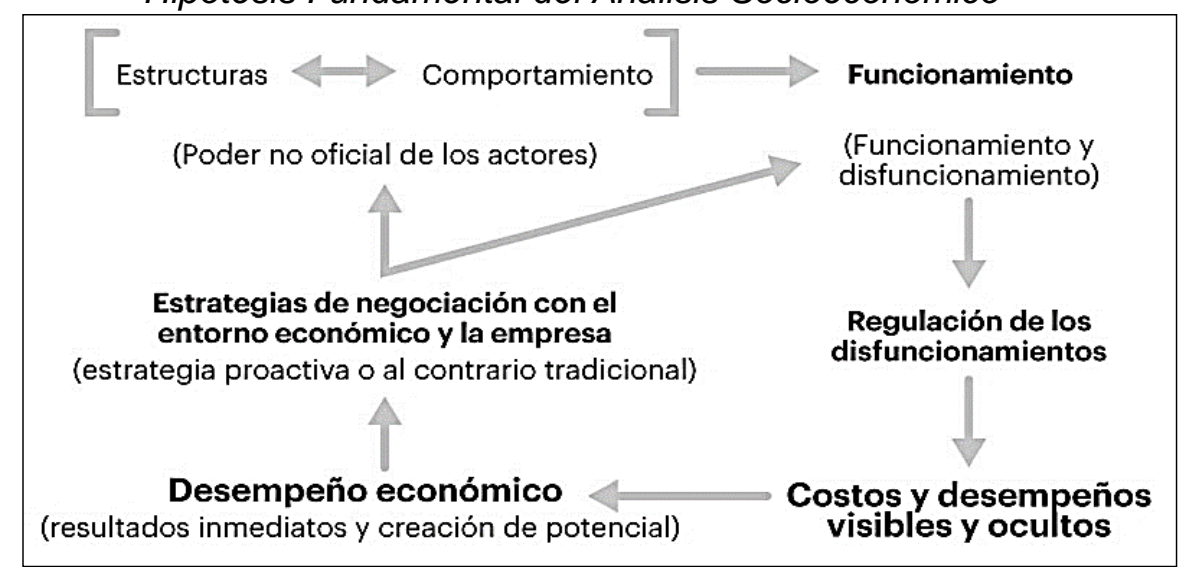

Fuente: Elaboración propia (2020), basado en Savall et al. (2008).

De manera oficial, se puede datar el surgimiento del enfoque en los años setentas, el cual tiene sus antecedentes en el contraste que hace Savall respecto a los resultados de las teorías clásicas en un contexto moderno, tomando como ejemplo a Taylor con la organización científica o Fayol con la teoría de la administración. El principal problema que se menciona es la naturaleza dicotómica de los mismos, los cuales asignaban una ponderación excesiva al concepto económico o al social, sin embargo, Savall et al. (2008)
La hipótesis retoma dos conceptos de vital importancia para el estudio: los disfuncionamientos y su relación con los costos ocultos. Los primeros son "el resultado del poder no oficial de los actores que interactúan ante las estructuras de la empresa o de la organización" (Savall et al., 2008, p. 135) y representan la diferencia entre el funcionamiento ideal 0 esperado y el funcionamiento real obtenido por los colaboradores. Los disfuncionamientos son clasificados en seis esferas 0 familias: condiciones de trabajo, 
organización del trabajo, comunicación-coordinaciónconcertación, gestión del tiempo, formación integrada e implementación de la estrategia, siendo esta última la de mayor trascendencia para el actual estudio.

Respecto a los costos ocultos, se refieren a aquellos costos que no cuentan con un nombre determinado, un monto calculado, ni un sistema de control específico respecto de su naturaleza. Representan "la expresión del poder informal de los asalariados de la empresa que se expresa a través de comportamientos disfuncionales" (Savall et al., 2008, p. 30), es decir, son la consecuencia económica de un disfuncionamiento (Brand, Vivanco, \& González, 2017). Se agrupan en seis componentes con la intención de evaluarlos financieramente: sobretiempos, sobresalarios, sobreconsumos, no producción, no creación de potencial y riesgos (Savall et al., 2009).
La importancia de identificarlos, surge del impacto que tienen como una reserva de desempeño y recursos para la empresa, al grado que si la entidad logra reducir su incidencia podría autofinanciarse, redireccionando el destino de los recursos para convertirlos en una inversión, puesto que se ha identificado que el monto promedio de estos costos es semejante al pago de la masa salarial (Savall et al., 2008).

Posteriormente, se complementó la hipótesis con la intención de identificar las variables del desempeño económico, marcando también los cinco indicadores de los costos ocultos (ausentismo, accidentes y/o enfermedades de trabajo, rotación de personal, no calidad y falta de productividad directa) en la representación de una empresa mediante el trébol de cuatro hojas (Figura 2), donde además se exponen los tipos de estructuras y comportamientos y su relación con los disfuncionamientos, lo cual impacta directamente en el desempeño económico de la empresa (Brand et al., 2017).

Figura 2.

Trébol de cuatro hojas del diagnóstico socioeconómico

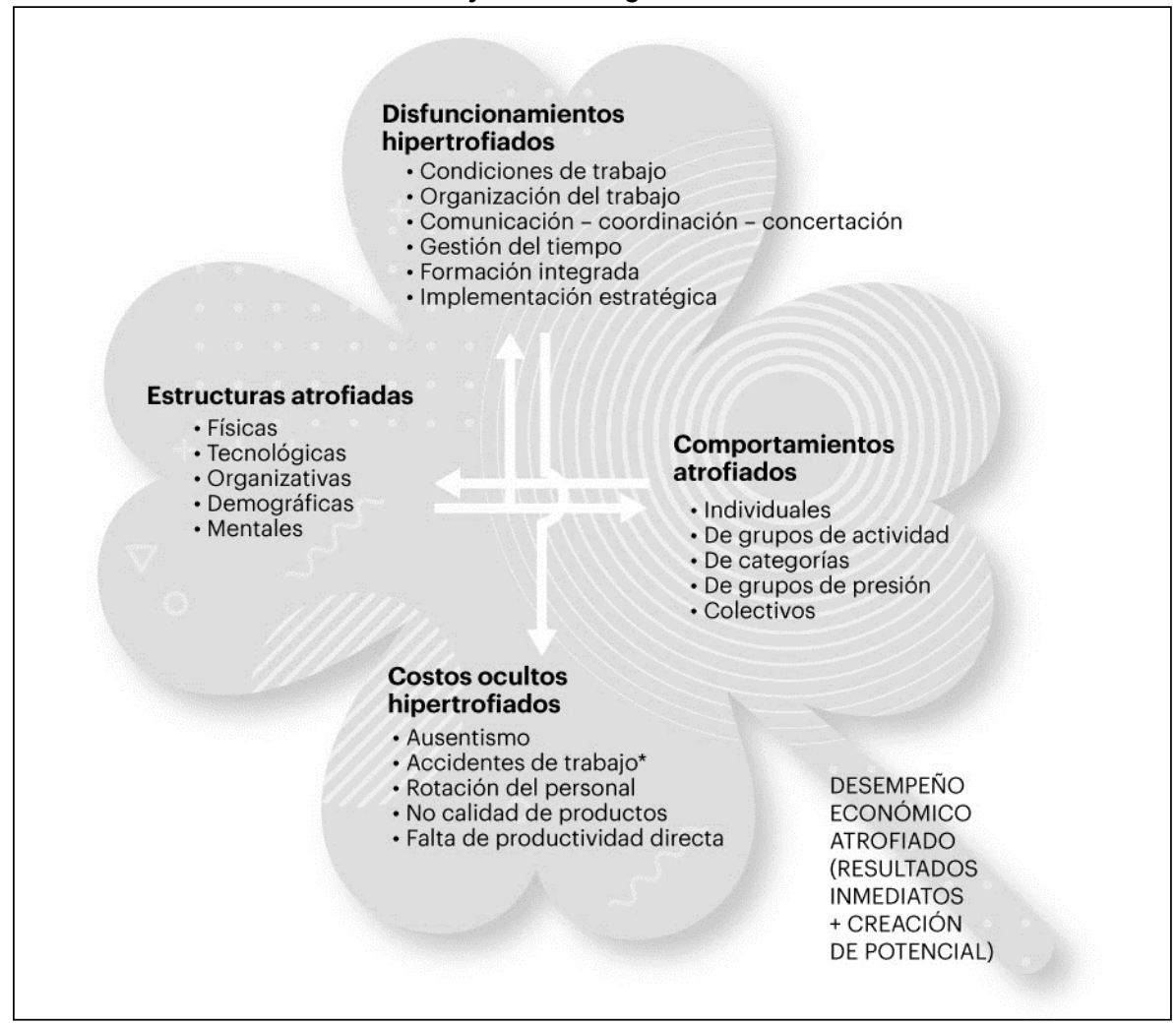

Fuente: Elaboración propia (2020), basado en Savall et al. (2008). 
(Figura 3): i) Eje del proceso de cambio, donde se establecen sus cuatro etapas de desarrollo; ii) Eje de herramientas de gestión, que marca el conjunto de herramientas propias del modelo que permiten llevar a cabo el proyecto; y iii) Eje de decisiones políticas y estratégicas, relacionado directamente con el proceso de negociación de los actores y la gestión del cambio a largo plazo (Parra y Peña, 2014).
"La doble intervención vertical y horizontal, conjunta, permite a los mandos intermedios aplicar la estrategia de forma óptima y encontrar soluciones tendientes a resolver los problemas identificados" (Savall et al., 2008, p. 77).

Figura 3.

\section{Ejes de la Gestión Socioeconómica}

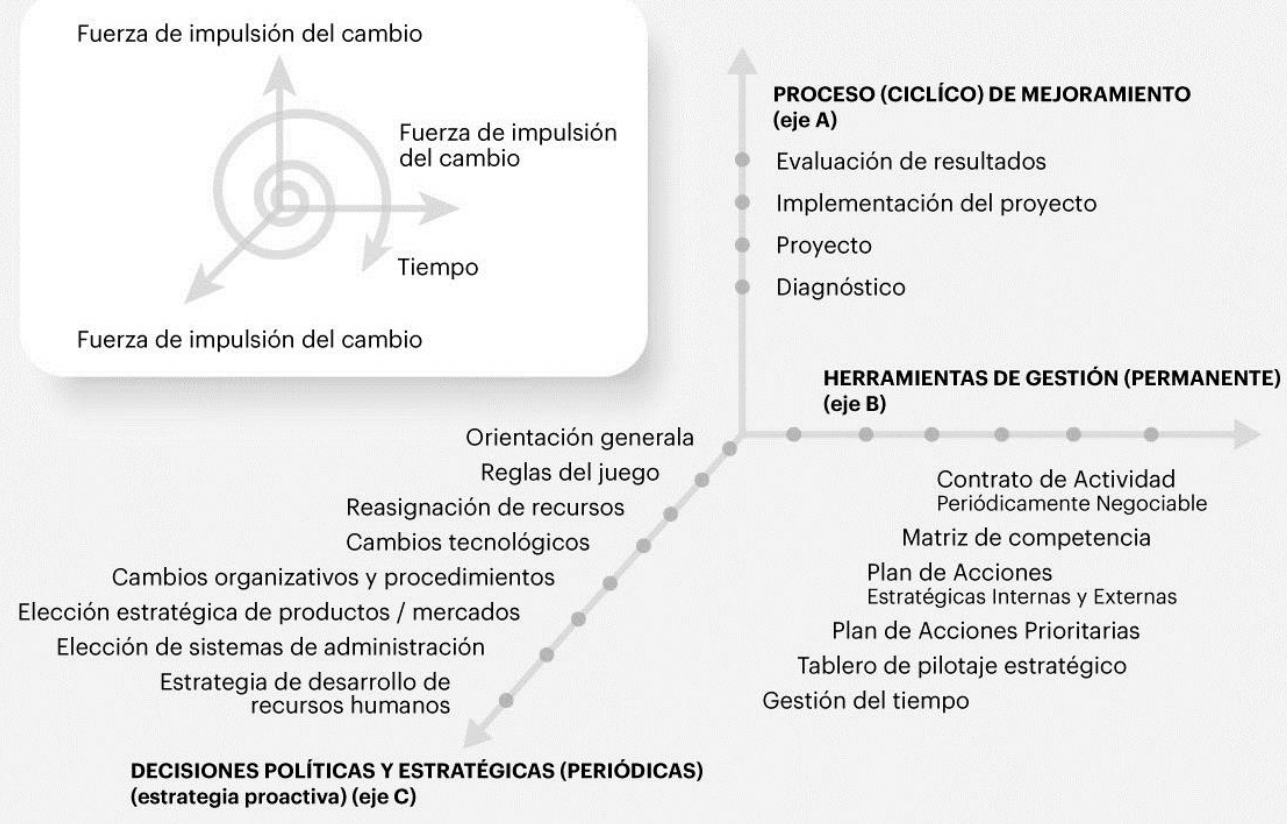

Fuente: Elaboración propia (2020), basado en Savall et al. (2008).

La implementación del MSE busca la integración de todas las áreas y niveles jerárquicos de la empresa, actuando de manera horizontal con los directivos y ejecutivos por área, así como de manera vertical con operativos y mandos medios, por lo cual se apoya del llamado método horizontal-vertical u HORIVERT (Brand et al., 2017).
Por otra parte, la estrategia representa el conjunto de medios o recursos para alcanzar los objetivos de la organización, tomando en cuenta los riesgos y la incertidumbre (Francés, 2006). Ésta corresponde a la manera en que una empresa se propone lograr sus objetivos, siguiendo un plan previamente definido pero que no garantiza el éxito contundente (Guadalupe, 2014). 
Por lo tanto, en el MSE la implementación de la estrategia "comprende la elaboración, realización y evaluación periódica de las acciones que permiten alcanzar los objetivos estratégicos internos y externos de la empresa" (Savall et al., 2008, p. 165).

Savall y Zardet (2009) mencionan que las herramientas para presentar las estrategias de mejora son, en general, poco elocuentes o contrastan en una dicotomía sobre presentar una débil formalización escrita o contar con una planificación demasiado compleja para su ejecución. Debido a lo anterior, el MSE plantea un conjunto de herramientas de gestión basadas en procedimientos más heurísticos de acuerdo con la complejidad que el entorno exige (Eje $B$ de la Figura 3). De ese colectivo se ha elegido el Plan de Acciones Estratégicas Internas y Externas (PAEINTEX), que es una lista de las actividades estratégicas que la empresa emprenderá en un mediano plazo, de tres a cinco años, jerarquizadas por importancia que servirá como referencia para directivos y mandos medios para identificar las actividades con valor, fomentando la mejora continua y reduciendo las restricciones del entorno (Savall et al., 2008).

\section{METODOLOGÍA:}

La intervención socioeconómica en empresas y organizaciones, permite realizar un diagnóstico de su situación actual para buscar identificar los disfuncionamientos en un inventario que sea relacionado con los costos ocultos que originan y, de esta manera, poder expresar el impacto que tienen en términos financieros.

Para clasificar adecuadamente cada disfuncionamiento identificado en el diagnóstico, se subdividen en 14 códigos o subgrupos dentro de la Implementación estratégica, que son: a) Orientación estratégica, b) Autores de la estrategia, c) Desagregación y organización de la puesta en práctica, d) Herramientas para puesta en práctica, e) Gestión del personal, f) Motivación y liderazgo, g) Rotación del personal, h) Inventarios, i) Implementación de política de compra y venta, $j$ ) Sistemas de remuneración y falta de prestaciones, $\boldsymbol{k}$ ) Calidad del producto y/o servicio, I) Cultura organizacional, $m$ ) Evaluación de desempeños inadecuada y n) Ausencia de Dirección.

En principio, el MSE se conforma de cuatro etapas (las cuáles se aprecian en el Eje A de la Figura 3), sin embargo, el estudio sólo desarrolla las dos primeras: el diagnóstico y la implementación, los cuales fueron realizados tanto a nivel directivo como operativo en todas las áreas (método HORIVERT). En la primera etapa se creó el vínculo con la organización, presentando el proyecto y la metodología. Para el diagnóstico de la empresa se recopiló información mediante la aplicación de entrevistas semiestructuradas a los actores, observación directa de la operación en el sitio y el análisis documental de los estados financieros para elaborar el inventario de disfuncionamientos sobre la implementación de la estrategia.

Con estos datos se generó un "efecto espejo" que pretende refleja fielmente los puntos de oportunidad con énfasis en las posibles mejoras y la necesidad de cambio interno a los actores de la empresa (Brand et al., 2017), iniciando la presentación con los niveles directivos y mandos medios.

En el caso de las entrevistas, para su aplicación fue necesario el diseño de un guion enfocado en obtener información principalmente de los disfuncionamientos correspondientes a la implementación de la estrategia y dirigido a todos los niveles de la empresa, de hecho, el alcance fue de la totalidad de directivos, mandos medios y del $90 \%$ de operativos, obteniendo la participación de 16 de los 17 colaboradores de la empresa, divididos en siete sesiones individuales y tres grupales (tres operativos por cada una).

Para la evaluación financiera de los disfuncionamientos, fue necesario calcular el valor del Nano PIB, que representa el valor perdido de un trabajador en una hora, (Pérez, 2012). El indicador se obtiene restando los costos variables a las ventas y dividiendo la diferencia entre el número de horas esperadas y remuneradas en el año, información contenida en los estados financieros revisados durante el análisis documental. Con el Nano PIB se pudo estimar la incurrencia de costos ocultos en cada disfuncionamiento junto con sus componentes e indicadores económicos correspondientes.

Posteriormente, se procedió con la segunda etapa: la propuesta del proyecto socioeconómico acorde con los 
puntos de oportunidad detectados en la implementación de la estrategia de la empresa, la cual se presenta mediante un PAEINTEX que integra cinco ejes estratégicos de acción, ordenados de acuerdo con el impacto financiero estimado en relación con los costos ocultos. Durante su elaboración, se contó con la participación de actores referentes a todos los niveles de la organización, siendo que los directivos influyeron más en el diseño de los objetivos y los operativos en la generación de las acciones específicas.

En concordancia con el enfoque cualimétrico de la investigación, el estudio se compone de un análisis cualitativo que culmina con el inventario de disfuncionamientos, seguido por la estimación de los costos ocultos en la parte cuantitativa, finalizando con el análisis comparativo de los estados financieros y los resultados obtenidos.

\section{RESULTADOS:}

Partiendo del análisis de la información recabada durante la fase diagnóstica, de acuerdo con la metodología del modelo, y su procesamiento con AtlasTi®, se logró obtener el inventario de disfuncionamientos relacionados con la implementación de la estrategia en la empresa estudiada. Gracias a la aplicación de entrevistas se obtuvieron un total de 181 frases testimonio después de filtrar las quejas: 109 frases de directivos y mandos medios sintetizadas en 26 ideas fuerza (que representan la noción principal de los enunciados) y 72 correspondientes a nivel operativo agrupadas en 25 ideas fuerza.

\section{Tabla 1.}

\section{Síntesis Frases Testimonio}

\begin{tabular}{|c|c|c|c|}
\hline \multicolumn{4}{|c|}{ Implementación de la estrategia } \\
\hline Códigos & Directivos & Operativos & Totales \\
\hline 1. Orientación estratégica & 16 & 5 & 21 \\
\hline 2. Autores de la estrategia & 5 & 3 & 8 \\
\hline 3. Desagregación y organización de la puesta en práctica & 8 & 2 & 10 \\
\hline 4. Herramientas para puesta en práctica & 4 & 3 & 7 \\
\hline 5. Gestión del personal & 13 & 12 & 25 \\
\hline 6. Motivación y liderazgo & 14 & 7 & 21 \\
\hline 7. Rotación del personal & 3 & 0 & 3 \\
\hline 8. Inventarios & 2 & 5 & 7 \\
\hline 9. Implementación de política de compra y venta & 7 & 2 & 9 \\
\hline 10. Sistemas de remuneración y falta de prestaciones & 9 & 7 & 16 \\
\hline 11. Calidad del producto y/o servicio & 11 & 10 & 21 \\
\hline 12. Cultura organizacional & 8 & 10 & 18 \\
\hline 13. Evaluación de desempeños inadecuada & 7 & 3 & 10 \\
\hline 14. Ausencia de Dirección & 2 & 3 & 5 \\
\hline Total & 109 & 72 & 181 \\
\hline Porcentaje & $60 \%$ & $40 \%$ & $100 \%$ \\
\hline
\end{tabular}

Fuente: Elaboración propia (2020) con base en las entrevistas aplicadas. 
Una vez compiladas las frases testimonio, se clasificaron en los 14 subgrupos considerados, como se puede apreciar en la Tabla 1, donde los códigos con mayor frecuencia pertenecen a Orientación estratégica, Gestión del personal, Motivación y liderazgo, así como Calidad del producto y/o servicio, las cuales en conjunto representan el casi el $50 \%$ del total de frases, entendiendo que la frecuencia de las frases es distinta al impacto del costo oculto en que pueden incurrir.

Continuando con los datos de la misma tabla, se aprecia que hay una mayor participación de directivos que han opinado más con respecto a la orientación estratégica y situaciones relacionadas con factores motivacionales, es decir, que tienen mayor pericia para localizar las fallas en la producción de los platillos y la atención al cliente que, en ocasiones, es minimizada o pasa desapercibida para el resto del equipo. Por parte de los operativos, los disfuncionamientos más discutidos han sido sobre la cultura organizacional. En el caso particular de los disfuncionamientos atribuidos a la gestión del personal, todos los niveles expresan su descontento por el manejo que se ha llevado del recurso humano en la empresa, lo cual incide directamente en el aumento de costos ocultos y la reducción de desempeños.
Al contrastar las ideas fuerza se puede apreciar que hay una convergencia en 19 de ellas entre los niveles, es decir, que coinciden y perciben los mismos disfuncionamientos, siendo las más representativas aquellas relacionadas con las ventas y atención al cliente, la ausencia de estrategias efectivas, la falta de herramientas administrativas en la empresa, la inadecuada gestión del personal y su motivación, el incumplimiento de las metas y que no existan indicadores para evaluar la operación objetivamente. En contraparte, las ideas fuerza que divergen entre los directivos y los operativos son 14, las cuales son percibidas bajo diferentes contextos y se refieren sobre todo a una mala definición de modelo de negocios, la discordancia de criterios para la operación, la falta de incentivos, la elevada rotación de personal, los problemas en abastecimiento e inventarios, los limitados resultados de la publicidad y la ausencia de políticas de compra.

Con la intención de ayudar visualmente a la interpretación de los datos, se generaron nubes de palabras como la presentada en la Figura 4, que corresponde a aquellas que aparecen con mayor frecuencia en las frases testimonio vinculadas con la implementación de la estrategia, como: empresa, personal, ventas, cliente, liderazgo, indicadores, estrategias, reglamento, jefes, sanciones, motivación, reglamento, atención, productos y atención.

Figura 4.

Nube de palabras: Implementación de la estrategia

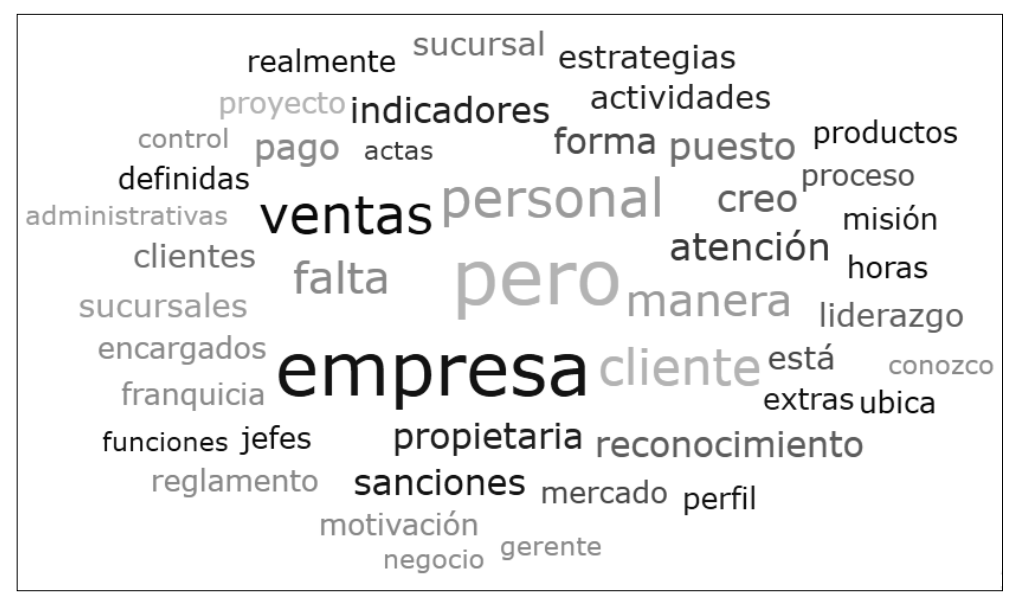

Fuente: Elaboración propia (2020) usando AtlasTi@. 
Asimismo, mediante el análisis de las coocurrencias detectadas con respecto a las frases testimonio, se reveló una mayor interrelación entre dos pares de subgrupos. En primer lugar, la conexión entre Motivación y liderazgo con los Sistemas de remuneración y fata de prestaciones, sustentada en la ausencia de un sueldo que integre bonos o incentivos que reconozcan o promuevan la consecución de resultados y estén alineados a los objetivos de la empresa. En segundo término, se relacionan la implementación de políticas de compra y venta con la Calidad del producto y/o servicio, entendiendo que la interrupción en la cadena de suministros afecta directamente y de manera importante la producción de los productos, así como el cumplimiento de la promesa de servicio que se hace a los clientes, impactando negativamente en las expectativas y la satisfacción de los mismos.

El análisis financiero parte de la estimación del Nano $\mathrm{PIB}$, que resultó con un valor de $\$ 41.90$ para la empresa estudiada, valor muy cercano al estandarizado por el ISEOR para empresas mexicanas, que es de $\$ 50.00$ (Pérez, 2012). A partir del inventario de disfuncionamientos y el Nano PIB, fue posible estimar los costos ocultos para la empresa intervenida, vinculando las consecuencias financieras con las fallas en la operación normal de la entidad, es decir, ligando los aspectos sociales con los financieros como lo marcan Savall y Zardet (2009). Asimismo, durante la estimación se calculó la masa salarial anual que sirvió como referente para la comparación de las cifras, la cual asciende a $\$ 840,946.80$.

Los costos ocultos anuales fueron calculados con relación a sus componentes e indicadores de acuerdo con ocho principales disfuncionamientos: a) Conflictos en la asignación de funciones $(\$ 190,624.23)$, que se ven reflejados en la desmotivación y la falta de control del personal, aunado a la indefinición de los puestos y sus actividades correspondientes; b) Presión laboral constante $(\$ 175,960.83)$, en conjunto con el deterioro del clima laboral, la programación de horarios no es eficiente para las necesidades de la empresa; c) Retardos del personal $(\$ 20,424.02)$, además de impactar disfuncionamientos como la organización del trabajo y la gestión del tiempo, se detectó que no hay un reglamento oficial que ayude a reducir los retardos o sancione estas incidencias; d) Procurar actividades sin valor agregado (\$96.527.08), este rubro se relaciona con el gasto de recursos e inversión de tiempo que conlleva realizar actividades que no generan valor a la empresa, por lo que se desaprovecha el talento del personal; e) Elevada rotación de personal (\$22,791.12), sobre todo en el área de panadería, donde la vacante se ofrece al menos dos veces por año, generando sobretiempos debido al reclutamiento e inducción, no producción durante los días que se requiere de personal y aumenta el riesgo de errores y estrés por aumentar la carga laboral de los colaboradores restantes; f) No diseñar los productos con orientación al cliente $(\$ 26,142.75)$, el no analizar el mercado ni dar seguimiento a los clientes produce que los productos no sean diseñados bajo criterios objetivos, además de causar pérdidas en los ajustes de las recetas; g) Inconsistencias en la calidad del producto $(\$ 169,179.29)$, la principal herramienta para corroborar el impacto de este punto son las encuestas de satisfacción, que revelan quejas sobre las porciones, los montajes, las temperaturas, la atención en servicio - los tiempos de entrega, aunado a deficiencias internas como la falta de estandarización de los procesos y protocolos de servicio; h) Planificación ineficiente $(\$ 33,516.35)$, que provoca repartición inequitativa de trabajo, fallas en los procedimientos e incumplimiento en los tiempos previstos.

Las estimaciones previas, fueron sintetizadas en el Modelo general de cálculo de costos ocultos, mostrado en la Tabla 2, cuyo monto total equivale a $\$ 753,165.67$ al año, donde la mayor incidencia por parte de los componentes está en la Falta de productividad directa con $\$ 496,628.49$. Por lo tanto, el total de costos ocultos relacionados con disfuncionamientos en la implementación de la estrategia representa el $87 \%$ de la masa salarial anual, los cuales se concentran principalmente en el área productiva, con el $78 \%$ repartido en dos indicadores: No producción $(\$ 308,159.20)$ y Sobretiempos $(\$ 266,974.47)$. 
Tabla 2.

Modelo General de Cálculo de Costos Ocultos Aplicado

\begin{tabular}{|c|c|c|c|c|c|c|c|}
\hline \multicolumn{8}{|c|}{ Modelo general de cálculo de costos ocultos aplicado } \\
\hline \multicolumn{4}{|c|}{ Sobrecargas (Costos Históricos) } & \multicolumn{4}{|c|}{ No producción (costos de oportunidad) } \\
\hline & $\begin{array}{l}\text { Sobre- } \\
\text { salarios }\end{array}$ & $\begin{array}{l}\text { Sobre- } \\
\text { tiempos }\end{array}$ & $\begin{array}{c}\text { Sobre- } \\
\text { consumos }\end{array}$ & $\begin{array}{c}\text { No } \\
\text { producción }\end{array}$ & $\begin{array}{c}\text { No } \\
\text { creación } \\
\text { de } \\
\text { potencial }\end{array}$ & Riesgos & $\begin{array}{c}\text { Total } \\
\text { Indicadores }\end{array}$ \\
\hline Ausentismo & & & & $\$ 20,424.02$ & & & $\$ 20,424.02$ \\
\hline $\begin{array}{l}\text { Accidentes de } \\
\text { trabajo }\end{array}$ & & & & & & & $\$ 0.00$ \\
\hline $\begin{array}{c}\text { Rotación de } \\
\text { personal }\end{array}$ & & $\$ 16,087.85$ & & $\$ 6,703.27$ & & & $\$ 22,791.12$ \\
\hline No calidad & & $\$ 26,746.04$ & $\$ 4,512.00$ & $\$ 8,544.00$ & & $\$ 155,520.00$ & $\$ 195,322.04$ \\
\hline $\begin{array}{c}\text { Falta de } \\
\text { productividad } \\
\text { directa }\end{array}$ & & $\$ 224,140.58$ & & $\$ 272,487.91$ & & & $\$ 496,628.49$ \\
\hline $\begin{array}{c}\text { Total } \\
\text { Componentes }\end{array}$ & $\$$ & $\$ 266,974.47$ & $\$ 4,512.00$ & $\$ 308,159.20$ & $\$$ & $\$ 155,520.00$ & $\$ 735,165.67$ \\
\hline
\end{tabular}

Terminado el diagnóstico se procedió con la segunda etapa, elaborando el proyecto de mejora con base en el análisis de la información obtenida. La propuesta se presenta mediante un PAEINTEX (Tabla 3), estructurado en ocho ejes estratégicos los cuales se ordenan de manera jerárquica descendente respecto a los costos ocultos relacionados, es decir, de acuerdo con los disfuncionamientos que cada eje busca resolver, se determinó el monto de costos que impactan y los más altos tienen los primeros puestos.
Finalmente, se entregaron los resultados a la empresa con la intención de brindarle las herramientas necesarias para atender los puntos de mejora y ejecutar las acciones estratégicas necesarias, principalmente con respecto a la reestructuración de la cultura organizacional, mejorar las condiciones de trabajo y normalizar los procesos para poder garantizar la calidad de los productos y la calidez del servicio.

Tabla 3.

Síntesis del Plan de Acciones Estratégicas Internas y Externas

\begin{tabular}{|c|c|c|}
\hline Ejes Estratégicos & Objetivos Estratégicos & Costo oculto relacionado \\
\hline I. Reestructuración de la cultura organizacional & $\begin{array}{l}\text { Evaluación del desempeño } \\
\text { Filosofía organizacional } \\
\text { Delegación de funciones }\end{array}$ & $\$ 211,048.25$ \\
\hline II. Mejoramiento de las condiciones de trabajo & $\begin{array}{c}\text { Integración y motivación } \\
\text { Canales de comunicación } \\
\text { Bienestar de los trabajadores }\end{array}$ & $\$ 198,751.95$ \\
\hline $\begin{array}{l}\text { III. Garantizar la calidad de los productos y del } \\
\text { servicio }\end{array}$ & $\begin{array}{c}\text { Normalizar procesos } \\
\text { Calidad en el servicio } \\
\text { Innovación organizacional }\end{array}$ & $\$ 195,322.04$ \\
\hline $\begin{array}{l}\text { IV. Aumento y desarrollo de las capacidades y } \\
\text { competencias }\end{array}$ & $\begin{array}{c}\text { Competencias } \\
\text { Conocimiento organizacional }\end{array}$ & $\$ 96,527.08$ \\
\hline V. Planificación efectiva de actividades & $\begin{array}{c}\text { Planeación estratégica } \\
\text { Control y toma de decisiones }\end{array}$ & $\$ 33,516.35$ \\
\hline
\end{tabular}

Fuente: Elaboración propia (2020). 


\section{CONCLUSIONES:}

La primera consideración al concluir debe orientarse a las limitaciones que presenta un estudio enfocado sólo en una de las seis familias de disfuncionamientos, implementación de la estrategia en este caso, puesto que el impacto social (181 frases testimonio compiladas) y el económico (costos ocultos estimados por $\$ 753,165.67$ ) se presentan como una parte del total potencial de desempeños y costos ocultos de la empresa intervenida, por lo que el diagnóstico de su contexto debe ser analizado bajo ese criterio de parcialidad.

Atendiendo la consideración previa, la implementación de la estrategia es uno de los grupos de disfuncionamientos que tienen un impacto importante en empresas de alimentos y bebidas, como muestra el estudio de Chávez (2017), que se encarga del diagnóstico a una Pyme restaurantera en Aguascalientes. En este caso se estimaron costos ocultos por un monto de $\$ 753,165.67$, de los cuales el componente con la mayor incidencia es la Falta de productividad directa con $\$ 496,628.49$, situación que coincide con otros estudios como el de Chávez (2017) o Villanueva (2014). En cuanto a los indicadores, la mayor carga de los costos se ubica en No producción $(\$ 308,159.20)$, relación que se comparte con los estudios de Chávez (2017), Villanueva (2014) o la propuesta de mejora para una empresa de la industria restaurantera de Ordoñez (2019).

Partiendo del monto de la masa salarial anual para la empresa, que es de $\$ 840,946.80$, se puede analizar comparativamente que los costos ocultos significan el $87 \%$ de ésta. Entonces, si una sola esfera de disfuncionamientos impacta de esta manera a la empresa, se puede apreciar que éstos costos pueden ser incluso mayores a la nómina considerada (Savall et al., 2008). Esta comparación pone en evidencia el posible autofinanciamiento, que se lograría al redireccionar los esfuerzos y recursos mal utilizados para convertirlos en inversión que agregue valor en la operación, sobre todo en el área productiva, donde se ha detectado la mayor incidencia de las desviaciones.

Por lo tanto, se concluye que la falta de consecución de objetivos esperados por parte de la empresa estudiada encuentra su origen en la insuficiencia e ineficiencia de las prácticas actuales que ha efectuado para controlar su operación, así como el diseño e implementación estratégica. Además, falta de sensibilidad y coordinación de los responsables al momento de sociabilizar las estrategias con todo el equipo. Por lo cual se le recomienda a la empresa analizar con mayor detalle puntos de oportunidad como la motivación, el diseño de objetivos y su alineación con la estrategia, cuidar la calidez en la atención al cliente y la calidad de los productos, así como revisar el sistema de remuneración del personal.

En este tenor, se observó que la mayor parte de los disfuncionamientos identificados se ubican 0 relacionan con el área de producción, por lo que el PAEINTEX realizado propone distintas acciones estratégicas principalmente con enfoque en mejorar las condiciones, la competitividad, la planificación y la calidad del servicio. Lo anterior se realizó con la intención de otorgar a la empresa una herramienta útil para la toma de decisiones con base en el diagnóstico generado y el autoconocimiento, recordando la integración de conceptos teóricos con la ejecución práctica propia del MSE, cuyo éxito en su implementación es corresponsabilidad de todos los actores.

Finalmente, un punto de oportunidad detectado para estudios posteriores surge en torno a la contingencia sanitaria a nivel internacional que ha afectado a todas las industrias, entre ellas la restaurantera. Las medidas de adaptación requeridas por los negocios de alimentos y bebidas para mantener las ventas se han orientado al servicio para llevar con los protocolos necesarios (Deloitte, 2020), aspecto que conlleva costos ocultos relacionados con los insumos necesarios o la infraestructura requerida, los cuales no fueron estimados al momento de terminar el estudio.

\section{REFERENCIAS:}

Brand, V., Vivanco, J., \& González, M. (2017). El modelo socioeconómico como moderador del desempeño organizacional en la Pequeña y Mediana Empresa familiar mexicana. Estudio de caso en Aguascalientes, México. Vértice Universitario(76), 29-40. 
CANIRAC (2016). Todo sobre la mesa: Dimensiones de la industria restaurantera. Documento de sitio web. México: www.canirac.org.mx. Recuperado el 07 de marzo de 2020, de https://canirac.org.mx/images/notas/files/TO DO\%20SOBRE\%20LA\%20MESA\%20BANN $\mathrm{ER}(1) . p d f$

Chávez, A. (2017). Elaboración de un diagnóstico financiero a través del método socioeconómico de una Pyme restaurantera en Aguascalientes [Tesis de Maestría]. México: Universidad Autónoma de Aguascalientes.

Deloitte. (2020). Restaurarte ante el COVID-19: Riesgos e implicaciones potenciales para la industria de restaurantes. [Webcast]. México: Deloitte S-Latam, S.C. Obtenido de https://canirac.org.mx/images/notas/files/Del oitte\%20Webcast_compressed.pdf

Francés, A. (2006). Estrategia y planes para la empresa con el cuadro de mando integral. México: Pearson Educación de México.

García, N. (2016). Estrategias para detectar y reducir los costos ocultos en el área de producción de una empresa productora de esencias $y$ colorantes a través del modelo socioeconómico de las organizaciones [Tesis de Maestría]. Ciudad de México: Instituto Politécnico Nacional.

González, F., \& Ganaza, J. (2015). Fundamentos de economía de la empresa. Madrid: Ediciones Pirámide.

Guadalupe, N. (2014). Plan Estatégico para el Restaurante Luna Bruja en la Paz, B.C.S. [Tesis de Maestría]. La paz, Baja California Sur, México: Instituto Tecnológico de La Paz.

ILPES/CEPAL. (2009). Manual de Planificación Estratégica e Indicadores de Desempeño en el Sector Público. [Manual]. CEPAL.
INEGI. (2016). Censos Económicos 2014. La industria restaurantera en México. México: Instituto Nacional de Estadística y Geografía.

INEGI. (2020). Comunicado de prensa número 305/20. México: Instituto Nacional de Estadística y Geografía.

LFT. (2019). Ley Federal del Trabajo. México: Diario Oficial de la Federación.

Ordoñez, J. (2019). Propuesta de un plan de mejora administrativa aplicando el modelo del management socioeconómico en una Pyme del estado de Aguascalientes [Tesis de Maestría].México: Universidad Autónoma de Aguascalientes.

Parra, J. \& Peña, Y. (2014). La teoría de los costos desempeños ocultos: una aproximación teórica. Cuadernos de Contabilidad, 15(39), 725-743

Pérez, C. (2012). Proyecto para mejorar el desempeño socioeconómico de la empresa COTSA: Un enfoque en las estructuras organizacionales [Tesis de Maestría]. México: Universidad Autónoma del Estado de Hidalgo.

Savall, H., \& Zardet, V. (2009). Ingeniería estratégica. Un enfoque socioeconómico. México: Universidad Autónoma Metropolitana.

Savall, H. Zardet, V., \& Bonnet, M. (2008). Mejorar los desempeños ocultos de las empresas a través de una gestión socioeconómica (Segunda ed.). Italia: OIT-ISEOR.

Villanueva, C. (2014). Diagnóstico financiero mediante el Método Socioeconómico (Caso práctico de 2 Pymes de la industria restaurantera del Estado de Aguascalientes) [Tesis de Maestría]. México: Universidad Autónoma de Aguascalientes. 